\title{
Learning Computational Methods for Partial Differential Equations from the Web
}

\author{
André Jaun ${ }^{1}$, Johan Hedin ${ }^{1}$, Thomas Johnson ${ }^{1}$, Michael Christie ${ }^{2}$, \\ Lars-Erik Jonsson ${ }^{3}$, Mikael Persson ${ }^{4}$, and Laurent Villard ${ }^{5}$ \\ 1 Alfvén Laboratory, Royal Institute of Technology, SE-100 44 Stockholm, Sweden, \\ jaun@fusion.kth.se, Web-page: http://pde.fusion.kth.se \\ ${ }^{2}$ Center for Educational Development, Chalmers, SE 41296 Göteborg, Sweden \\ ${ }^{3}$ Unit for Pedagogy and Didactics, University, SE 41296 Göteborg, Sweden \\ ${ }^{4}$ Electromagnetics, Chalmers Institute of Technology, SE 41296 Göteborg, Sweden, \\ ${ }^{5}$ CRPP, Ecole Polytechnique Fédérale, CH-1015 Lausanne, Switzerland
}

\begin{abstract}
A course has been developed to learn computational methods from the wel[ 1$]$ and has been tested with postgraduate students from remote universities. Short video conferences or video recordings provide an overview and introduce more detailed studies with numerical experiments in Java-powered course notes. This enables every participant to work at his own pace and to develop his intuition for finite differences, finite elements, Fourier, Monte-Carlo and Lagrangian methods. Assignments are carried out in a regular web browser and are compiled into web pages where the students explain with their own words, equations and programs how to derive, implement and run computational schemes. Our experience shows that the technology is rapidly acquired from templates, using practical examples for the advection, diffusion, Black-Scholes, Burger, Korteweg-DeVries and Schrödinger equations.
\end{abstract}

\section{Introduction}

Computational methods are part of the problem-solving skills that need to be mastered by professionals working in a quantitative field. At an advanced level, excellent textbooks generally provide a robust mathematical foundation for one specific approach; they however miss the overview and examples, which are necessary at an introductory level to choose the right method and implement a practical solution. Convinced that the Internet technology can be of great value in this context, we created a problem-based learning environment where the acquisition of knowledge is motivated by well defined tasks; in parallel, we switched from a teaching-centered to a learning-centered course where the students explore the material with the focus, order and pace they choose by themselves. This paper describes the learning method we tested 1997-2000 with summer courses involving 10-20 participants geographically dispersed around Stockholom and Göteborg. Together with the educational material, it is our nice human experience and the encouraging results that we would like to share here with you.

\footnotetext{
${ }^{1}$ http://pde.fusion.kth.se
} 


\section{A Distance Learning Setup}

The course begins with an announcement in the schools' mailing list 2 outlining the subject. A link to the course notes on the web and former student projects enables potential participants to judge if the content is aligned with their target curriculum. This way of proceeding reflects the current trend towards a free market for university courses and is well adapted to offering teaching services outside traditional school boundaries, such as sister universities and private companies.

Every morning during two weeks, the students are encouraged to attend a lecture in one of the video-conference rooms and may download a video recording from the Internet. Both are optional and serve as an introduction to a second active learning phase where the knowledge is acquired with experiments in the Java-powered course notes. Most of the time is spent carrying out the exercises in a regular browser, form where they are automatically compiled into Web pages. Once they are ready, the students submit their solutions electronically for correction to a (human) teacher. Discussion forums exploit the ability of quick learners to answer simple questions from peers and enable the teacher to focus on the problems where his expertise is most useful and precious. A third week is generally necessary to fulfill all the requirements and is well spent in building up a working knowledge in a variety of methods by solving concrete problems.

Being active researchers in theoretical plasma physics, it is clear that we could only devote a limited amount of time to the entire project. How much can be achieved in a total of six months teaching over three years, how large is the technology burden and how useful are the electronic tools in this context was largely unknown. Moreover, is it reasonable to expect students to visit universities and even pay for courses if the notes are readily accessible from the Internet? Our experience shows that those students who have the possibility to attend classes locally or through video-conference still do so for the stimulation and discussions they get from the teacher and peers. Those who cannot strongly value the flexibility of delivering exercises when and from where they like and yet to benefit from a close personal supervision with corrections from a teacher.

\section{Classroom or Video Lectures Provide an Overview}

Classroom lectures introduce web page equivalents of the course notes [1] and are broadcast by video conference or (RealVideo) recordings to remote participants. The lessons are short (30-40 minutes) to keep the attention of the audience; every now and then, a short quiz stimulates lively discussions locally before the conclusions are shared in a more orderly manner between remote classrooms. The JBONE applet (Java Bed for ONE dimensional problems) is used to test new schemes directly in the web browser; this adds an unprecedented animation and interactivity to the lecture and is extremely valuable when comparing the numerical properties of different time evolution schemes. Menus select the equation

\footnotetext{
2 students97@engineering.myschool.edu,phd-students@yourschool.edu
} 
(advection diffusion, Burger's shock waves, Korteweg-De Vries solitons, BlackScholes options, Schrödinger, etc), the initial condition (box, Gaussian, cosine, soliton, put option, wavepacket) and editable text fields control the parameters (velocity, diffusion, dispersion, time step, etc) directly in the web browser. A mouse click starts the simulation, making it extremely easy and convincing to illustrate for example the linear instability that occurs when the time step gets too large in an explicit finite difference advection scheme, or to show how the more subtle aliasing in spectral methods affects a non linear train of solitons.

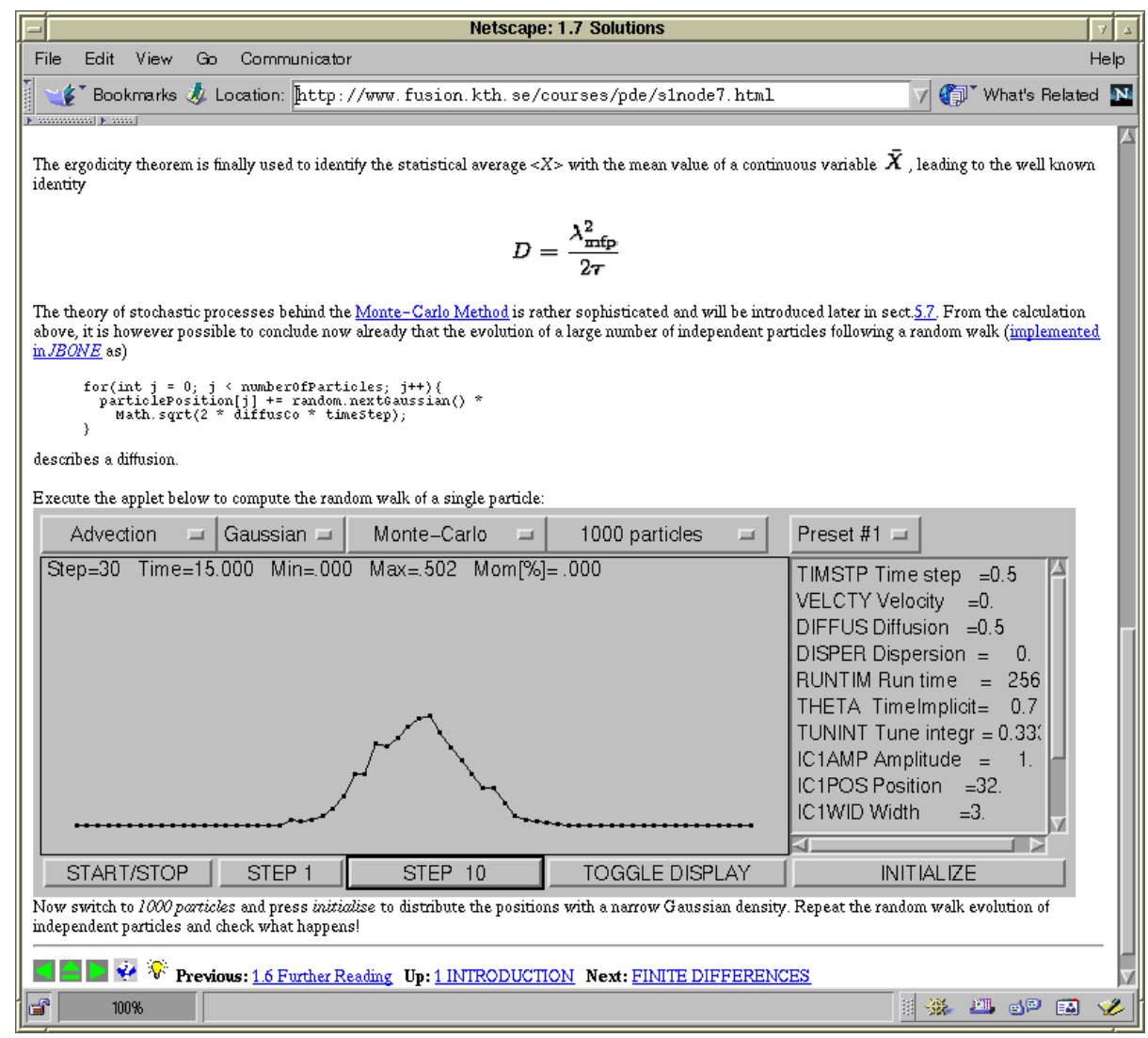

Fig. 1. Screen capture of the web browser displaying an analytical formula, the algorithm with a hyperlink into the source code and the JBONE applet - all after execution of the Monte-Carlo integration with 1000 particles to illustrate the connection between the motion of random walkers and diffusion. 


\section{Active Learning with Experiments from Home}

An advantage of using widespread, platform independent technology is that the students can reproduce and modify the demonstrations back in their office or directly from home. Repeating the line of thought from the classroom, the text and figures take the reader through series of analytical derivations that yield a computational scheme. Hyperlinks point to the relevant sections in the code and show how every algorithm has been implemented. Default parameters are preset to illustrate specific properties, but can be modified to verify if a topic has been correctly understood.

Example: From Brownian motion to diffusion. Both are fundamental in science and engineering and are often hard to understand for undergraduates. An analytical derivation of the RMS displacement $\sqrt{\left\langle\xi_{i}^{2}\right\rangle}$ in a particle's random walk connecting the diffusion coefficient to the square of the mean free path divided by the collision time $D=\lambda_{m f p}^{2} / 2 \tau_{c}$ might en-light a few, but is likely to loose a majority in the algebra. Using the Monte-Carlo evolution from the applet displayed in figure 1 it is simple to demonstrate first how the random motion of a single particle can be described with the Java code

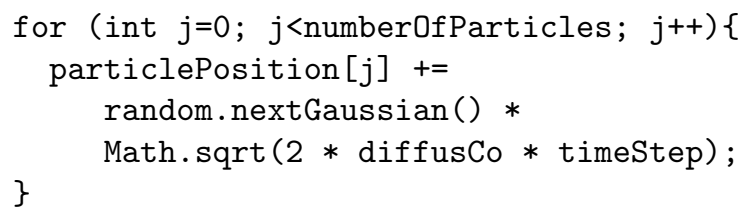

Distributing 1000 particles to approximate an initial Gaussian distribution, the same algorithm immediately shows what diffusion means. Change the initial condition to a cosine or a square box and it is equally rewarding to demonstrate how short wavelength modes decay more rapidly than long wavelengths.

Having part of the students studying away from the campus, is it possible to use the technology to stimulate personal interactions with the teacher and peers? Yes, better than we thought!

\section{Assignments Carried out Directly on the Web}

Exercises in the first session are designed to familiarize the students with the electronic publishing on the web; templates show how the building blocks are used in relevant schemes to assimilate $\mathrm{T}_{\mathrm{E}} \mathrm{X}$ and Java directly through the context. Teaching at an advanced level where copying is not an issue, we distribute a list of all the solutions web pages and let the students compare and discuss the results 
with each other. Our top pick of the best solution creates a healthy competition where everybody tries to become a member of a very exclusive list.

Part of the students choose to carry out an additional one week project, applying their favorite method to a topic of interest such as the Black-Scholes equation for a European call option, a tunable finite elements integration for the Schrödinger equation, a mesh refinement procedure, iterative solvers, etc. Given the small amount of time allocated for each project, the scope remains of course limited; by cross-checking each other's reports on the web, the students nevertheless get an overview of a rather broad range of applications.

The material. A single $\mathrm{T}_{\mathrm{E}} \mathrm{X}$ source generates both the printed course notes and the hyperlinked web pages. Running open software translators such as latex $2 \mathrm{html}$ [2], tth [3] and scripts embedded in a makefile, the static web material can effectively be produced with no additional cost to what is required to print the notes and slides. Writing the JBONE applet from scratch was quite an effort for the teachers, but the object-oriented language and the encapsulated structure of the code enables students with little programming experience to gradually modify existing schemes and add their own. A substantial amount of documentation (programming tree, keyword index) is created automatically using the javadoc utility, which is part of the standard Java development kit. An automatic download service has been set up for teachers and individuals who would like to use, modify and tailor our material for their specific needs.

\section{$6 \quad$ Working with Discussion Forums}

News groups or discussion forums prove to be an ideal tool allowing a sufficient number of participants to interact in a geographically distributed environment. Not only do students help and discuss with each other at virtually any time of the day and night, but the advice they get or provide is usually helpful and competent. Some supervision is required, but instead of answering a dozen times to the same question (often for organizational matters), the teacher can intervene once for an announcement and spend the rest of the time clarifying discussions that remain very informal and help the students to understand the subject in their own language. To encourage interactions between students and create the feeling of belonging to the same virtual classroom, we now reward relevant contributions as well as the assignments.

\section{Evaluation and Conclusion}

Three classes with 11,18,15 participants went through considerably different learning schemes since the first time the course was taught in a conventional manner (in 1997), introducing a problem based learning with the JBONE applet (1999), using video-conferences between two universities (2000) and allowing now for a distance learning at any time and from anywhere on the Web. We 
cannot really draw statistically significant conclusions about the effectiveness of each; anonymous evaluations and discussions with the students do nevertheless indicate that the largest benefits come the problem-based learning and the simple user interface. These are much more challenging to implement than hyper-linked documents, when firewalls, different computer platforms, versions of software and operating systems can quickly become a technology nightmare!

Templates provide the most efficient help for the electronic submission of the home assignments. This allows the questions asked in the discussion forums to deal mainly the computational aspects - the substance of the course. One participant proposed to create a discussion group reserved for alumni to maintain valuable contacts after graduation.

In its full electronic form, the course clearly requires a well-maintained web server, which is generally administered by an assistant in a university. Because of the ask once, answer to all nature of the discussion forums, assistants can however be employed very efficiently and the overall teaching load is finally similar to a conventional setup.

Some flexibility is required from both the lecturer and the students in order to exploit the new possibilities and work around the weaknesses of a course taught at a distance. Our experience however shows that the pedagogical content is by no means reduced if the technology can be used to support a problem-based learning context with a forum allowing the students to discuss and understand the material with their own words. The enthusiasm from all the participants including the teaching assistants is a very gratifying experience and should be an additional encouragement to try similar experiments elsewhere.

\section{Acknowledgements}

This work is supported in part by the Summer University of Southern Stockholm (SUSS) and Ericsson.

\section{References}

1. Jaun A., Hedin J., Johnson T., Numerical Methods for Partial Differential Equations. TRITA-ALF-1999-05 (1999), http://pde.fusion.kth.se

2. Drakos N., Text to Hypertext conversion with LaTeX2HTML. Baskerville 3 (1993) 12, http://cbl.leeds.ac.uk/nikos/tex2html/doc/latex2html/latex2html.html

3. Hutchinson, I., TTH: a TeX to HTML translator. http://hutchinson.belmont.ma.us/tth/ 